\title{
Why we edit
}

\author{
All articles in Nature Reviews Physics are extensively edited in-house before publication. What do \\ we do, and why?
} 9 Good editing,
like good writing,
always has the
reader in mind
One of the first experiences of a new editor in a Nature Reviews journal is to write a Research Highlight. After putting hours of thought into every word and phrase, the editor confidently sends the piece to her colleagues. The document is swiftly returned covered in tracked changes and comments, and she wonders if her hard work was for nothing - no one likes being edited. But whether you're a tenured professor, postdoc, professional science writer or member of the editorial team, every sentence you write for Nature Reviews Physics will be scrutinized before publication.

Good editing, like good writing, always has the reader in mind. At Nature Reviews Physics, we want our articles to be accessible to a broad physics audience, not just specialists who are already familiar with the topic. Our goal is that any physics graduate student should be able to understand and make use of our content. The most important way we make our articles more accessible is to edit their structure. A good structure guides the reader through the text and figures, anticipating their questions. It makes the word 'clearly' unnecessary, because every step of the argument is clear.

For authors who have spent weeks thinking about and writing a piece, it is easy to become so familiar with their articles topic and message that it is impossible to see any structural flaw. There might be a key piece of information that is assumed rather than stated, unexplained jumps between topics or narrative that backtracks rather than flows. Or perhaps the article has several authors and the sections don't fit together smoothly. Such features are often more visible to someone coming at the text with fresh eyes. Referees can pick up on such details, but we do not expect our referees to put hours into suggesting edits for the text of a review - this is our role, as professional editors. We have the expertise and time to dedicate to develop the text with our broad readership in mind. Because we focus on structure, our line-editing goes well beyond copyediting, which deals primarily with grammar and consistency.

As editors, we are also in a position to spot concepts that need a little more explanation for a novice reader. We keep in mind terms that can mean something different to the different communities our journal serves.
Often physics terms are words that mean very different things in everyday English, but even in a physics context, writers can fall into the trap of using words confusingly. For example, in quantum mechanics, 'unitarity' refers to algebraic properties of operators, whereas for ultra-cold atomic gases it refers to a separation of length scales such that only density is relevant. For readers already familiar with the topic of a review, the meaning of the word is obvious, but readers who are new to a field can be tripped up by a word used in an unfamiliar way. A little extra context or explanation can be a big help, and as editors we often suggest adding it. Symbols can be particularly confusing - is $D$ a diffusion constant or the dimensionality of the system? - and we ensure that all symbols are defined and used consistently. We edit figures, to make sure that every figure contributes to the story and that plots that come from different third-party sources use consistent style and notation. Our strict house style has subtleties that may seem pedantic, but that can improve readability by removing extraneous abbreviations and avoiding quirks of the English language. In addition, because our audience is international, we avoid unusual idioms and prefer simple phrasing; conversely, our editorial support means that authors can contribute their ideas and expertise regardless of their English ability. Finally, many submissions are about a 'plethora' of 'unique' and 'novel' phenomena that promise to lead to a 'Holy Grail'. We would love all our articles to be 'paradigm-shifting', but we ruthlessly cut hyperbole.

Editing is a two-way process. We want to retain our authors' voices in the articles they write, and we want to hear if our suggested changes have missed the intended meaning of the text. We discuss with our authors how to identify the best way of conveying difficult concepts and avoiding possible confusion. No matter what shape an article is in when it arrives on our desk, it can always be improved by a constructive dialogue between authors and editor. As Stephen King said, "[No] writer will take all of his or her editor's advice; for all have sinned and fallen short of editorial perfection. Put another way, to write is human, to edit is divine." 1. King, S. On Writing: A Memoir of the Craft (Simon and Schuster,
2000). 